\title{
Preface to the special issue: commutativity of algebraic diagrams
}

\author{
RALPH MATTHES and SERGEI SOLOVIEV \\ Institut de Recherche en Informatique de Toulouse (IRIT), \\ C.N.R.S. et Université Paul Sabatier (Toulouse III), \\ 118 route de Narbonne, F-31062 Toulouse Cedex 9, France
}

Received 19 October 2011

The problem of the commutativity of algebraic (categorical) diagrams has attracted the attention of researchers for a long time. For example, the related notion of coherence was discussed in Mac Lane's homology book Mac Lane (1963), see also his AMS presidential address Mac Lane (1976). Researchers in category theory view this problem from a specific angle, and for them it is not just a question of convenient notation, though it is worth mentioning the important role that notation plays in the development of science (take, for example, the progress made after the introduction of symbolic notation in logics or matrix notation in algebra). In 1976, Peter Freyd published the paper 'Properties Invariant within Equivalence Types of Categories' (Freyd 1976), where the central role is played by the notion of a 'diagrammatic property'. We may also recall the process of 'diagram chasing', and its applications in topology and algebra. But before we can use diagrams (and the principal property of a diagram is its commutativity), it is vital for us to be able to check whether a diagram is commutative.

The use of diagrams in category theory is so well established a practice that we might ask what progress can still be expected in this domain beyond the 'instrumental' use of diagrams to obtain some new technical result. When we organised the workshop CAMCAD on the commutativity of algebraic diagrams (Toulouse, 16-17 October 2009), our first thoughts were of the general methods for verifying commutativity, rather than simple uses of diagrams as notation, and we wanted to bring together researchers working on applications of categorical diagrams in mathematics (algebra and topology) and computer science (models and metamodels, rewriting systems and higher order languages).

Nine talks were presented at the workshop. The main topics covered in the talks were: graphs and graphical reasoning, also in connection with quantum computations (Lucas Dixon and Alex Kissinger, and Pierre Rannou); graph rewriting and diagram transformations (Yves Guiraud, Philippe Malbos and Samuel Mimram); typed and untyped computations (Damien Pous); paths in categories and bifibrations (Robert Hein and Francois Lamarche); dependency of diagrams (Antoine El Khoury, Sergei Soloviev, Laurent Méhats and Mark Spivakovsky); and diagrammatic syntax in general (Andrei Rodin). For more details, see http://www.irit.fr/CAMCAD09/.

We expected there to be more talks on the connection between proofs and categorical diagrams (it is well known that morphisms in free categories with structure can be represented using proofs in certain logical systems, and the applications of proof theory to 
category theory based on this fact have a long and honourable history). We also expected to have presentations on implementation issues. In fact, the majority of talks were devoted to the use of combinatorial methods and rewriting.

The post-proceedings of the workshop presented in this Special Issue of Mathematical Structures in Computer Science were open to non-participants and solicited much more detailed research papers. Out of six submitted papers, three were selected ${ }^{\dagger}$.

Most of the papers presented in this issue adopt a combinatorial approach. Shuffles and concatenations in the construction of graphs by Kosta Došen and Zoran Petric investigates the uses of shuffles and concatenation in the treatment of graphs and partial orders in relation to categorical coherence questions. Coherence in monoidal track categories by Yves Guiraud and Philippe Malbos develops homotopical methods based on rewriting in higher-dimensional categories and applies them to prove concrete coherence results. Properties of co-operations: diagrammatic proofs by Pierre Rannou develops an alternative approach to computations in bialgebras, which is based on diagram rewriting.

This Special Issue highlights only a few aspects of the research activities concerning the commutativity of algebraic diagrams. The variety of work in progress on implementation issues (with varying degrees of ambition and/or practicability) shows that good systems for symbolic processing of diagrams are of vital importance and greatly needed. The absence of sufficiently powerful, efficient and universal systems may be one of the obstacles to more rapid progress in categorical methods in the research environment, where there is a growing role played by computer supported proof development.

In this context, we should mention the promising attempts in the development of computer-assisted processing of diagrams including: GDCT (Graphical Database for Category Theory) by Robert Rosebrugh et al. at Mount Allison University; a project by Michael J. Healy at the University of New Mexico; the web page devoted to the interactive category theory demonstrations by Jocelyn Ireson-Paine; the work in LISP by Alexandar Bakic; and developments in Haskell by Vasili I. Galchin. We cannot give an exhaustive list here, but it is notable that these attempts are at the moment mostly limited to categories generated by finite graphs and basic constructions of category theory. However, it would be interesting, for example, to develop systems for the treatment of diagrams in categories with structure such as Closed Categories of different types.

The development of such systems would be a key development in the approach to many problems in category theory, foundations of mathematics and computer science, and it will help bridge many of the gaps separating researchers in different domains. One of the possible obstacles to progress in this research is a well-known technical difficulty arising for many coherence problems (which may be seen as problems of commutativity of large classes of diagrams). But a more important obstacle may be the problem of communication between researchers working in adjacent domains. To see the problems in a larger context would be very helpful.

\footnotetext{
$\dagger$ On the basis of the referee reports, we rejected two papers and suggested considerable modifications to a third. However, the revisions to the latter paper were not completed in time for publication, so it is not included here, but we hope it will appear separately in a later issue of MSCS.
} 
The contribution of our workshop and this Special Issue may be viewed as an initial step and a guide for this more practical implementation task going beyond basic category theory constructions - mostly on the algorithmic side. As illustrated by this introduction, this field will also profit from bringing together researchers working in many adjacent domains.

\section{Acknowledgements}

Our thanks go to the participants of the workshop, the authors of the submissions to this issue, whether they were accepted or not, to the anonymous referees for their efforts and to Giuseppe Longo, the Editor-in-Chief of Mathematical Structures in Computer Science, for having given us the opportunity to prepare this Special Issue.

\section{References}

Freyd, P. (1976) Properties Invariant within Equivalence Types of Categories. In: Heller, A. and Tierney, M. (eds.) Algebra, Topology and Category Theories, Academic Press 55-61.

Mac Lane, S. (1963) Homology, Springer-Verlag.

Mac Lane, S. (1976) Topology and Logic as a Source of Algebra. Bulletin of the American Mathematical Society 82 (1) 1-40. 